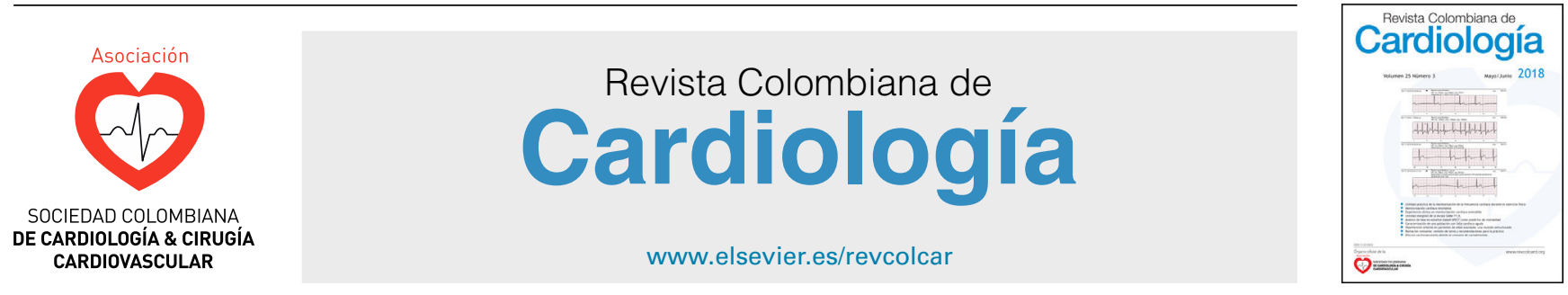

CARDIOLOGÍA PEDIÁTRICA - PRESENTACIÓN DE CASOS

\title{
Impresión 3D de rara patología congénita de aorta y vasos supraaórticos
}

\section{Susan Pumacayo-Cárdenas ${ }^{a, *}$, Gustavo Arias-Vela ${ }^{a}$ y Edgar Quea-Pinto ${ }^{b}$}

a Universidad Nacional de San Agustín, Arequipa, Perú

b Hospital Nacional Edgardo Rebagliatti Martins-Essalud, Lima, Perú

Recibido el 13 de febrero de 2018; aceptado el 20 de octubre de 2019

Disponible en Internet el 18 de abril de 2020

\section{PALABRAS CLAVE Impresión tridimensional; Divertículo; Síndromes del arco aórtico}

\section{KEYWORDS}

Three-dimensional printing;

Diverticulum;

Artic arch syndromes

\begin{abstract}
Resumen El divertículo de Kommerell es una anomalía de muy baja incidencia y prevalencia en la edad pediátrica. Se reporta el caso de un paciente de 11 años de edad, con diagnóstico de divertículo de Kommerell con arco aórtico derecho y origen anómalo de arteria subclavia izquierda desde la rama pulmonar izquierda a través de conducto arterioso persistente. Dado que es una anomalía cardiovascular compleja se decidió realizar un modelo impreso en 3D, el cual proporcionó una mejor comprensión de su distribución espacial, tamaño y forma real, como si fuera una pieza de anatomía patológica. Este modelo ayudó en la toma de decisiones, planificación y seguridad de la ejecución de una posible cirugía cardiaca. Este es el primer reporte de caso de este tipo de anomalía, así como el primer prototipo cardíaco impreso en modo tridimensional realizado en Perú para el tratamiento de la misma.

(C) 2020 Sociedad Colombiana de Cardiología y Cirugía Cardiovascular. Publicado por Elsevier España, S.L.U. Este es un artículo Open Access bajo la licencia CC BY-NC-ND (http:// creativecommons.org/licenses/by-nc-nd/4.0/).
\end{abstract}

\section{3-Dimensional impression of a rare congenital disease of aortic and supra-aortic vessels}

Abstract Kommerell's diverticulum is an anomaly of very low incidence and prevalence in paediatrics. A case is presented of an 11 year-old patient with a diagnosis of Kommerell's diverticulum with a right aortic arch and a left subclavian artery of anomalous origin from the pulmonary branch through a patent ductus arteriosus. Given that this was a complex cardiovascular anomaly, it was decided to make a print model in 3-D. This provided a better understanding of its spatial distribution, size, and real shape, as it was a histopathology piece. This model

\footnotetext{
* Autor para correspondencia.

Correo electrónico: pumacayocardenas@gmail.com (S. Pumacayo-Cárdenas).
} 
helped in taking planning and safety decisions on any possible cardiac surgery. This is the first report of a case of this type of anomaly, as well as the first prototype of a cardiac print in 3-dimensional mode, performed in Peru.

(c) 2020 Sociedad Colombiana de Cardiología y Cirugía Cardiovascular. Published by Elsevier España, S.L.U. This is an open access article under the CC BY-NC-ND license (http:// creativecommons.org/licenses/by-nc-nd/4.0/).

\section{Introducción}

La impresión tridimensional (3D) es un proceso de fabricación innovador que permite la conversión de datos de imágenes 3D en "impresiones" físicas. En los últimos cinco años ha habido un notable aumento en los estudios publicados e informes de casos con aplicaciones de impresión $3 \mathrm{D}$ en cardiopatía congénita. En este artículo se presenta la aplicación de esta herramienta en una anomalía infrecuente de la aorta y de los vasos supraaórticos.

\section{Caso}

Paciente varón, de 11 años de edad, sin antecedentes patológicos de importancia, quien acudió por dolor torácico retroesternal leve, tipo opresivo, de presentación esporádica (una vez al mes), después de la ingesta de alimentos sólidos; negó síncope. Clase funcional I. Al examen físico: exploración cardiaca normal, presión arterial: brazo derecho 105/65 y en brazo izquierdo 95/60; leve disminución de pulso radial izquierdo (respecto al derecho) con llenado capilar adecuado; miembros superiores simétricos, trofismo adecuado. ECG en ritmo sinusal, en límites normales. La ecocardiografía reveló arco aórtico a la derecha y vasos supraaórticos de difícil evaluación; no se encontró ningún defecto congénito asociado. La tomografía confirmó arco ártico derecho con origen de los vasos supraaórticos, así: origen común de ambas carótidas (tronco bicarotídeo), seguido de subclavia derecha, y en el arco distal presenta dilatación tipo divertículo de Kommerell, con las siguientes medidas: base $14 \mathrm{~mm}$ de diámetro (figs. 1A, B) y $26 \mathrm{~mm}$ de diámetro, medido desde la pared aórtica opuesta hacia la punta del divertículo de Kommerell (fig. 1B). En la parte anterior y la derecha del divertículo se observa tráquea sin evidencia de compresión o desplazamiento; entre la tráquea y el divertículo cursa esófago vacuo (colapsado), aunque no llega a conformar un anillo vascular completo. Se observa arteria subclavia izquierda de pequeño calibre ( $2 \mathrm{~mm}$ de diámetro), con origen en la rama pulmonar izquierda (en posición de conducto arterioso). El trayecto de su segmento proximal es longitudinal, paralelo a la tráquea, levemente tortuoso y de curso anterior al divertículo, sin compresión del esófago ni de la vía aérea proximal y distal, ya que discurre lejano a ellos; sin embargo, después de dar origen a la arteria vertebral, recupera su calibre, similar a su contralateral (fig. 1C). Para precisar mejor esta anomalía y su abordaje se utilizó una nueva herramienta en el estudio de imágenes médicas, la “impresión en 3D” (figs. 2 y 3 ).
Este modelo $3 \mathrm{D}$ es una reconstrucción computarizada de los datos de imágenes (DICOM) superpuestos uno sobre otro para crear el objeto "completo", el cual derivó de la utilización del software 3 Dimensional Slicer 4.6. Las imágenes se convirtieron a un formato digital específico de estéreolitografía (figs. 2B y 3), que posteriormente se interpretó y procesó por el hardware, es decir, por la Impresora $3 \mathrm{~d}$ Replicator Z18 3 d Printer Makerbot. El tiempo aproximado que tomó el proceso de segmentación de la imagen y obtención del producto fue cinco horas, se usó poliamida 618 y se obtuvo como resultado el modelo impreso en tres dimensiones (fig. 3).

\section{Discusión}

El caso que se reporta es relevante debido que el divertículo de Kommerell con arco aórtico derecho constituye una anomalía congénita con una incidencia tan baja como del 0,05 al $0,1 \%$ en las series radiológicas ${ }^{1,2}$. Por otro lado, si se tiene en cuenta sólo la configuración del arco aórtico derecho con arteria subclavia izquierda aberrante (origen desde el conducto arterioso), también se tiene una prevalencia muy baja, 0,04 a 0,4\%, dos veces más frecuente en varones ${ }^{3,4}$.

Las alteraciones del desarrollo y las variaciones del arco aórtico pueden explicarse con base en la hipótesis del doble arco aórtico de Edwards. En pacientes con arco aórtico derecho con divertículo de Kommerell, como en el caso que se expone, esta teoría plantea que el arco dorsal izquierdo disminuye, hasta su desaparición, entre las arterias subclavia izquierda y carótida izquierda. Esto permite que la subclavia derecha se origine en el arco dorsal derecho remanente; en algunas ocasiones ambas carótidas llegarían a fusionarse a nivel de sus orígenes, formando el tronco bicarotídeo. El origen de la subclavia izquierda se explicaría por obliteración de la subclavia izquierda, causando su independencia del arco, con colateralización vía conducto arterioso, por la persistencia del sexto arco aórtico izquierdo. El doble arco aórtico a veces se confunde con el divertículo de Kommerell porque ambos causan disfagia lusoria. Sin embargo, estas dos condiciones deben diferenciarse porque el divertículo (divertículo de Kommerell) es un remanente del arco dorsal que ha perdido la continuidad del $\operatorname{arco}^{5,6}$.

El caso expuesto es importante por su baja frecuencia, y aunque existen diferentes clasificaciones del arco aórtico derecho y anomalías de los vasos supraaórticos, en cualquiera de ellas el arco aórtico derecho con origen de la arteria subclavia izquierda desde el conducto arterioso es muy raro. El divertículo de Kommerell generalmente es 

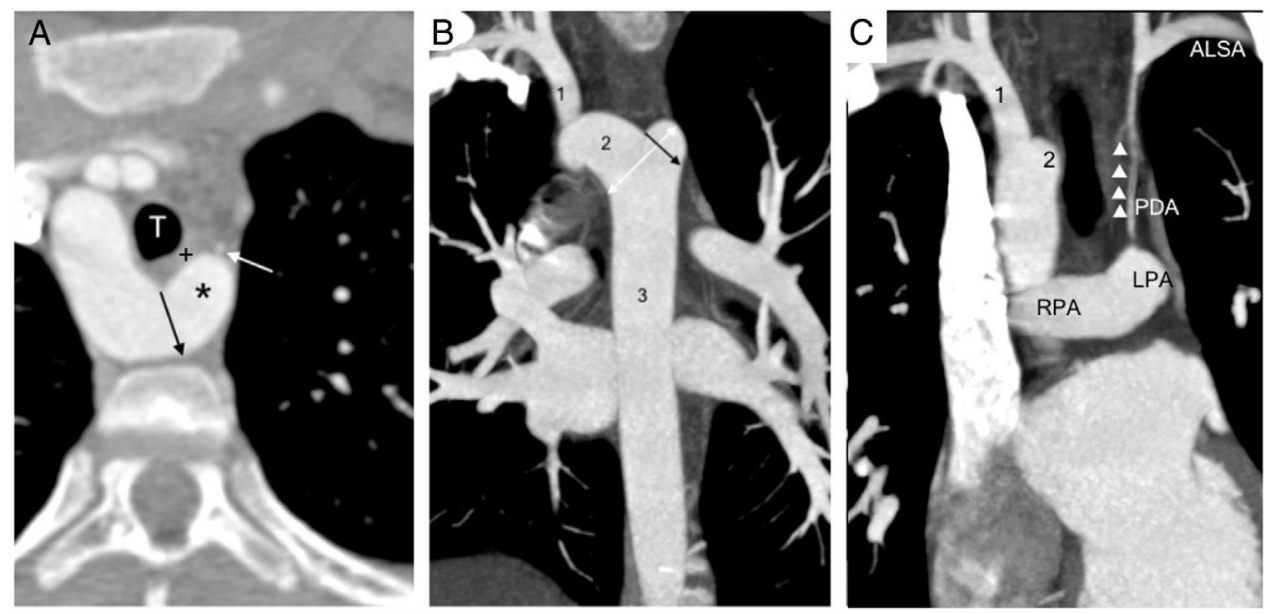

Figura 1 Tomografía axial computarizada. A. TAC plano axial; se aprecia arco aórtico a la derecha y divertículo de Kommerell ( $\left.{ }^{*}\right)$ con su orificio de entrada o base que mide $14 \mathrm{~mm}$ (flecha negra). En su parte anterior se observa subclavia izquierda de pequeño calibre (flecha blanca), esófago(+), tráquea(T). B. Se observa divertículo en vista coronal, el diámetro de su base (flecha negra) y el diámetro desde la pared aórtica opuesta a la punta del divertículo (flecha blanca de doble cabeza). C. Se muestra arteria subclavia izquierda aberrante (ALSA) con origen desde la rama pulmonar izquierda (LPA) a través del conducto arterioso (PDA), con pequeño calibre en su segmento proximal (triángulos), después del origen la vertebral normaliza su diámetro. (1) Arteria subclavia derecha. (2) Arco aórtico transverso. (3) Aorta descendente. (RPA) Rama pulmonar derecha.
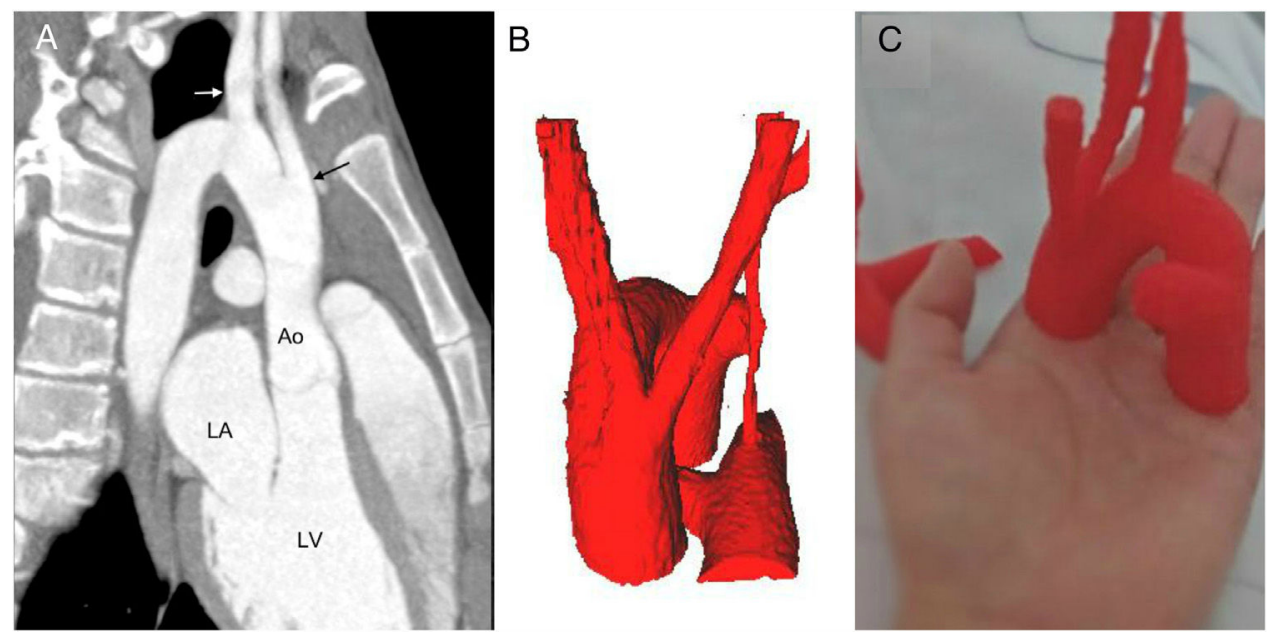

Figura 2 Proceso de conversión. A. TAC. Se muestra origen de los troncos supraaórticos: tronco común de carótidas (flecha negra) y subclavia derecha (flecha blanca). (Ao) Raíz aórtica. (LV) ventrículo izquierdo. B. Conversión a formato virtual digital del área de interés en $3 \mathrm{D}$ (específico de estéreo-litografía). C. Modelo impreso tridimensional.

asintomático, pero se sabe que es un factor que predispone para la formación aneurismática, la dilatación con compresión de la tráquea o el esófago, la disección y la ruptura. La arteria subclavia izquierda en niños presenta síntomas como resultado de la compresión lusoria o estructural por el divertículo. Los problemas respiratorios son más comunes que en los adultos debido a una mayor rigidez traqueal ${ }^{6,7}$. La sintomatología del caso consistió en dolor torácico asociado a disfagia esporádica aislada, debido a la compresión anterior del esófago a causa del divertículo de Kommerell, que formaba un anillo vascular incompleto, en el que la arteria subclavia izquierda no interviene por tener un trayecto independiente desde la rama pulmonar izquierda, alejado de la vía aérea y del esófago. El paciente no presentó síntomas de síndrome de robo de flujo de subclavia ni signos de alteraciones en la circulación distal, con adecuado desarrollo del miembro superior izquierdo, que podría explicarse por la presencia de circulación colateral suficiente desde los vasos supraaórticos hacia la arteria subclavia izquierda, que no se demostró en la tomografía por el campo de adquisición limitado (no se realizó completamente en la zona de los vasos supraaórticos) y probablemente por ser de vasos de pequeño calibre. Se decidió realizar intervención quirúrgica programada según el bajo riesgo de isquemia del brazo izquierdo, la ausencia de otros defectos cardiacos congénitos, el seguimiento de imágenes y la evolución de la sintomatología, ya que los síntomas y signos fueron aislados, hubo ausencia de compromiso de vía aérea, y por las características 

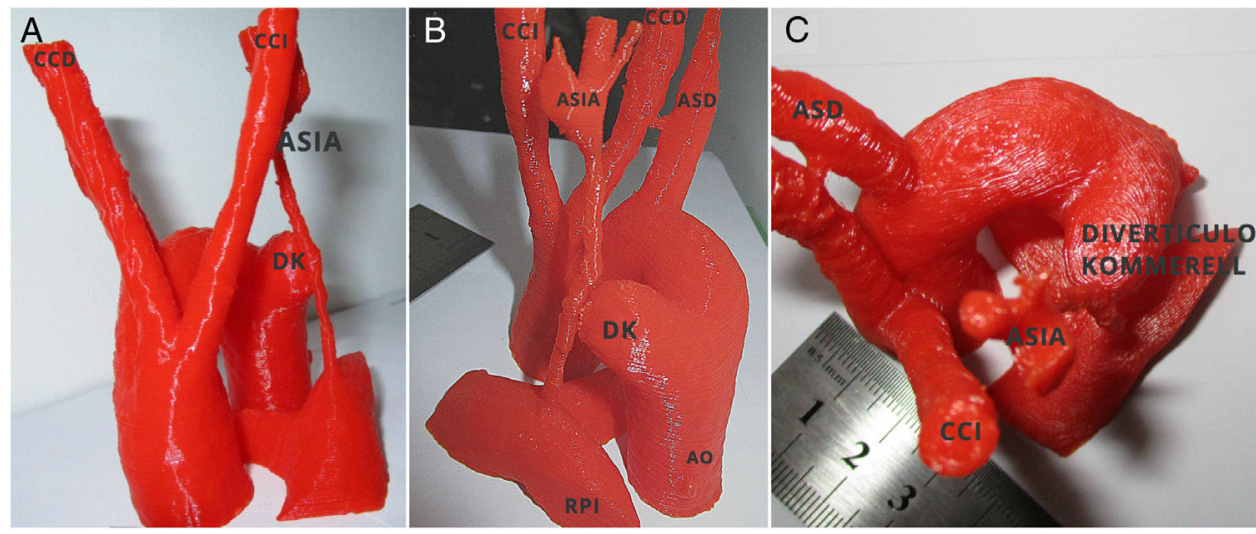

Figura 3 Modelo impreso en $3 \mathrm{D}$. A. Vista frontal de modelo $3 \mathrm{D}$. Origen común de ambas carótidas de aorta ascendente,DK y relación con ASIA. B. Vista posterior izquierda. Se observa de manera clara origen de ASIA de RPI y su relación con el divertículo. C. Vista superior. Muestra distribución anómala de vasos supraórticos, forma y tamaño exacto de DK. CCD: carótida común derecha. CCI: carótida común izquierda. ASD: Art. subclavia derecha. ASIA: Art. subclavia izquierda aberrante. AO: aorta descendente. DK: divertículo de Kommerell. RPI: rama pulmonar izquierda.

anatómicas del divertículo de Kommerell (que se describen más adelante).

El ADD asociado con el divertículo de Kommerell tiene riesgo del $19 \%$ al $53 \%$ de ruptura o disección, lo cual es potencialmente mortal. La indicación del tratamiento dependerá de la presencia de sintomatología, que se describió anteriormente, y por las características anatómicas del divertículo de Kommerell. Respecto al tipo de tratamiento existen varias opiniones, aunque el quirúrgico sigue siendo el que más se describe ${ }^{8-10}$. Algunos estudios recomiendan la resección del divertículo de Kommerell aneurismático (diámetro mayor que 1,5 veces el tamaño de la arteria subclavia), incluso en pacientes asintomáticos para prevenir disección o ruptura potencial ${ }^{8,11}$. Aunque el tamaño específico del divertículo para indicación quirúrgica no se ha definido claramente debido a los escasos datos disponibles se han propuesto las siguientes medidas: más de $50 \mathrm{~mm}$ de diámetro máximo desde la pared aórtica opuesta hacia la punta del divertículo de Kommerell ${ }^{11,12}$ y/o el diámetro del orificio de entrada al divertículo es mayor a $30 \mathrm{~mm}^{120,13}$. De acuerdo con estos tamaños, en el paciente las medidas fueron 26 y $14 \mathrm{~mm}$ respectivamente, que según los parámetros ya mencionados no reúnen criterios de intervención quirúrgica inmediata, si bien el enfoque quirúrgico, ya sea cirugía abierta o híbrido/endovascular, debe escogerse en función de las experiencias de cada centro ${ }^{13}$.

El uso de modelos impresos en 3D en el campo médico está en desarrollo y ha demostrado su impacto en la educación y la adquisición de habilidades del personal de salud (clínicos como quirúrgicos, por ejemplo en el entrenamiento de cirujanos cardiovasculares). Mediante ensayos controlados aleatorios se ha comparado el uso de modelos tridimensionales con los métodos tradicionales de aprendizaje, y se han hallado diferencias en términos de conocimientos, habilidades y satisfacción del profesional $^{4,14-16}$. Con este uso, como en nuestro caso, el paciente es el más beneficiado en todos los aspectos. Se habla, por ejemplo, de anticipación a complicaciones por un adecuado planeamiento, tiempos operatorios menores $y$, en el caso de adultos, de utilidad en la colocación de prótesis valvulares ${ }^{16-19}$. En este caso también se obtuvo mejor comunicación con los pacientes y familiares, ya que se puede realizar una explicación detallada sobre la anomalía, su abordaje terapéutico y los riesgos respectivos ${ }^{4,15,16}$.

Existen pruebas que apoyan que los modelos impresos en $3 \mathrm{D}$ son superiores al uso de imágenes $3 \mathrm{D}$ convencionales; es decir, se ha observado que las habilidades viso-espaciales humanas, como la "rotación mental" y la conceptualización $3 \mathrm{D}$, son limitadas y varían significativamente entre individuos. Así, las impresiones en $3 \mathrm{D}$ eliminan estas limitaciones y no dejan a la imaginación del observador ningún aspecto de la relación espacial de las estructuras cardiacas 3,19,20. Coincidimos con ellos ya que nuestro trabajo permitió entender las estructuras vasculares de una forma clara y real, equivalente a tener una pieza de anatomía patológica. Es importante destacar que las autopsias en nuestro medio son infrecuentes por aspectos socioculturales, así es que no se puede acceder al estudio de las cardiopatías congénitas con piezas anatómicas reales.

En el caso presentado es indudable que al obtenerse el modelo impreso $3 \mathrm{D}$ se dio un gran paso en la comprensión de las cardiopatías congénitas, y es el inicio del uso de nuevas herramientas tecnológicas. En Perú constituye el primer modelo impreso en $3 \mathrm{D}$ y el primer reporte de divertículo de Kommerell con arco aórtico derecho y arteria subclavia izquierda desde el conducto arterioso persistente.

\section{Conclusión}

El divertículo de Kommerell con arco aórtico derecho y arteria subclavia izquierda es una anomalía de muy baja frecuencia, que puede ser asintomática o tener manifestaciones de otros sistemas; sin embargo, el uso de un modelo impreso tridimensional ha constituido una herramienta útil en la comprensión de esta rara condición. Este caso es el primer reporte de este tipo de anomalía y de utilización de impresión $3 \mathrm{D}$ en nuestro medio. 


\section{Financiación}

\section{Autofinanciado.}

\section{Conflicto de intereses}

Ninguno.

\section{Bibliografía}

1. Tsukube T, Ataka K, Sakata M, Wakita N, Okita Y. Surgical treatment of an aneurysm in the right aortic arch with aberrant left subclavian artery. Ann Thorac Surg. 2001;71:1710-1.

2. Svensson L, Crawford E. Congenital anomalies of the aorta in adults. En: Svensson LG, Crawford ES, editores. Cardiovascular and Vascular Disease of the Aorta. Philadelphia: Saunders; 1997. p. 153-74.

3. Chen N, Zhu K, Zhang H, Sun X, Wang C. Three-dimensional printing guided surgery in complex aortic disease. J Thorac Dis. 2017;9:1639-43.

4. Saeed G, Neuzner J, Gradaus R. Double aortic arch and Kommerell's diverticulum. Asian Cardiovasc Thorac Ann. 2014;22:882-3.

5. Tanaka A, Milner R, Ota T. Kommerell's diverticulum in the current era: a comprehensive review. Gen Thorac Cardiovasc Surg. 2015;63:245-60.

6. Calderón J, Muñoz L, García J, Ramírez S, Patiño E, Buendía A. Diverticulum of Kommerell. Arch Cardiol Mex. 2005;75:451-4.

7. Cosío L, Ríos J, Peralta G, Cosío M. Arco aórtico derecho con subclavia izquierda aberrante que se origina de un divertículo de Kommerell Revisión actualizada de la literatura y presentación de un caso clínico. Acta Médica Grupo Angeles. 2014;12:76-84.

8. Kim KM, Cambria RP, Isselbacher EM, Baker JN, LaMuraglia GM, Stone JR, et al. Treatment of Kommerell diverticulum. Ann Thorac Surg. 2014;98:1347-54.

9. Edwards JE. Anomalies of the derivatives of the aortic arch system. Med Clin North Am. 1948;32:925-49.

10. Tsukube T, Ataka K, Sakata M, Wakita N, Okita Y. Surgical treatment of an aneurysm in the right aortic arch with aberrant left subclavian artery. Ann Thorac Surg. 2001;71:1710-1.
11. Ota T, Okada K, Takanashi S, Yamamoto S, Okita Y. Surgical treatment for Kommerell's diverticulum. J Thorac Cardiovasc Surg. 2006;131:574-8.

12. Idrees J, Keshavamurthy S, Subramanian S, Clair DG, Svensson LG, Roselli EE. Hybrid repair of Kommerell diverticulum. J Thorac Cardiovasc Surg. 2014;147:973-6.

13. Gould SW, Rigsby CK, Donnelly LF, McCulloch M, Pizarro C, Epelman M. Useful signs for the assessment of vascular rings on cross-sectional imaging. Pediatr Radiol. 2015;45: 2004-16.

14. Meier LM, Meineri M, Qua Hiansen J, Horlick EM. Structural and congenital heart disease interventions: the role of three-dimensional printing. Neth Heart J. 2017;25:65-75, http://dx.doi.org/10.1007/s12471-016-0942-3

15. Olejník P, Nosal M, Havran T, Furdova A, Cizmar M, Furdova A, et al. Utilisation of three-dimensional printed heart models for operative planning of complex congenital heart defects. Kardiologia Polska. 2017;75:495-501.

16. Byrne N, Velasco Forte M, Tandon A, Valverde I, Hussain T. A systematic review of image segmentation methodology, used in the additive manufacture of patient-specific $3 \mathrm{D}$ printed models of the cardiovascular system. JRSM Cardiovasc Dis. 2016;5, 2048004016645467. doi:10.1177/2048004016645467.

17. Kappanayil M, Koneti NR, Kannan RR, Kottayil BP, Kumar K. Three-dimensional-printed cardiac prototypes aid surgical decision-making and preoperative planning in selected cases of complex congenital heart diseases: Early experience and proof of concept in a resource-limited environment. Ann Pediatr Card. 2017;10:117-25.

18. Bartel T, Rivard A, Jiménez A, Mestres C, Müller S. Medical three-dimensional printing opens up new opportunities in cardiology and cardiac surgery. Eur Heart J. 2018;39:1246-54, http://dx.doi.org/10.1093/eurheartj/ehx016

19. Jaworski R, Haponiuk I, Chojnicki M, Olszewski H, Lulewicz P. Three-dimensional printing technology supports surgery planning in patients with complex congenital heart defects. Kardiologia Polska. 2017;75:185-7.

20. Meier LM, Meineri M, Qua Hiansen J, Horlick EM. Structural and congenital heart disease interventions: the role of three-dimensional printing. Neth Heart J. 2017;25:65-75, http://dx.doi.org/10.1007/s12471-016-0942-3 